\title{
Acquired optociliary shunt vessels in papilloedema
}

\author{
H. M. EGGERS* AND M. D. SANDERS \\ From the National Hospital for Nervous Diseases, Queen Square, London WC1N $3 B G$
}

SUMMARY Seven cases are presented in which prolonged papilloedema led to the development of acquired optociliary shunt vessels. These vessels may also be found with optic nerve tumours, particularly spheno-orbital meningiomas, optic nerve drusen, glaucoma, and after central retinal vein occlusion. Two patients had intracranial tumours, 4 benign intracranial hypertension, and one Crouzon's disease. Three had marked atrophic changes of the disc. The pathophysiology of the disc changes is discussed. The triad of long-standing poor vision, acquired optociliary shunts, and optic atrophy with blurred disc margins should not be regarded as specific for spheno-orbital meningioma.

Acquired optociliary shunt vessels present a rare and distinctive clinical appearance. There have been several reports of these vessels due to sphenoorbital meningiomas. ${ }^{1-3}$ These vessels have also been noted in association with optic nerve glioma, ${ }^{45}$ optic nerve arachnoid cyst, ${ }^{6}$ optic disc drusen, ${ }^{7}$ glaucoma, ${ }^{8-10}$ and after central retinal vein occlusion $^{511-13}$ There are some early references to the occurence of these shunt vessels in papilloedema ${ }^{14-16}$ but few modern documented cases. ${ }^{18}{ }^{18}$ We therefore present 7 cases of optociliary shunt vessels occurring with papilloedema.

\section{Patients and methods}

\section{CASE 1}

A 37-year-old housewife was admitted for increasing weakness of the left arm and leg of 2 years duration and several recent attacks of focal epilepsy. Her past history included migraine episodes for the past 6 years. The general physical examination was unremarkable. Neurological examination showed left pyramidal tract signs and diminished 2-point discrimination in the left hand. There was a loud bruit audible all over the skull. The ocular examination showed a corrected visual acuity of $6 / 6$, N6 in the right eye and 6/5, N5 in the left. There was a right relative afferent pupillary defect. Perimetry showed the visual field of the left eye to be full, but there was some constriction of the nasal field of the

*Present address: E. S. Harkness Eye Institute $635 \mathrm{~W}$. 165 St., New York, N.Y. 10032.

Correspondence to Mr M. D. Sanders. right eye. The optic discs had blurred margins and were somewhat pale. The right was paler than the left. There were small drusen-like bodies at the disc margins and tortuous optociliary shunt vessels on both discs. Fluorescein angiography in both eyes showed mild dilatation of the radial peripapillary capillary plexus overlying the disc and nearby retina and leakage of dye into the surrounding retina, confirming the presence of papilloedema (Fig. 1). The shunt vessels filled from the retinal circulation. Arteriography demonstrated a massive superficial arteriovenous malformation in the right middle cerebral artery territory.

\section{CASE 2}

A 37-year-old electrician was evaluated for diminished visual acuity in the left eye of indeterminate duration and 3 months of intermittent visual obscurations of both eyes. The obscurations were consistently brought on by running down stairs or bending over. The general and neurological examinations were normal except for bilateral papilloedema. Corrected visual acuity was $6 / 6$ in the right eye and 6/18 left. There was a left relative afferent pupillary defect. Perimetry showed the visual field of the right eye to be moderately constricted in a concentric pattern, and the visual field of the left eye to be markedly constricted, especially nasally. There was bilateral papilloedema with tortuous optociliary shunts and temporal pallor of the left disc (Fig. 2). Fluorescein angiography showed profuse dye leakage at both discs and demonstrated the shunt vessels in the left eye to fill from the retinal veins. At operation a colloid cyst of the third ventricle was removed. 


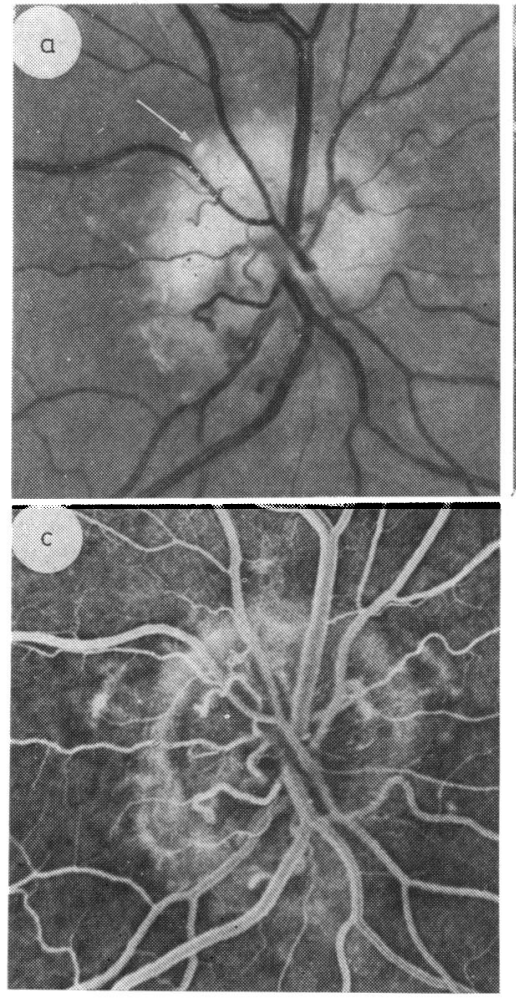

CASE 3

A 43-year-old executive presented with a 6-month history of progressive visual deterioration, predominantly of the left eye. The general and neurological examinations showed some blunting of the intellect, right sided hyposmia, and pathologically brisk reflexes of the left arm, but was otherwise unremarkable. The ocular examination showed a visual acuity of $6 / 5$ in the right eye and counting fingers at $1 \mathrm{~m}$ in the left. There was a left relative afferent pupillary defect. Perimetry showed a right inferior temporal defect extending to the enlarged blind spot, but not the midline, and a small temporal island of vision remaining in the left eye. There was bilateral chronic papilloedema with moderate atrophy of the left disc, which had an ectatic, tortuous shunt vessel. Fluorescein angiography showed leakage at the discs and demonstrated the shunt vessel to fill from the retinal circulation. At surgery a right subfrontal meningioma was removed. Repeat examination 11 days postoperatively showed the shunt vessel to have disappeared (Fig. 3).

CASE 4

A slightly obese 20-year-old female was evaluated for headaches. General physical and neurological

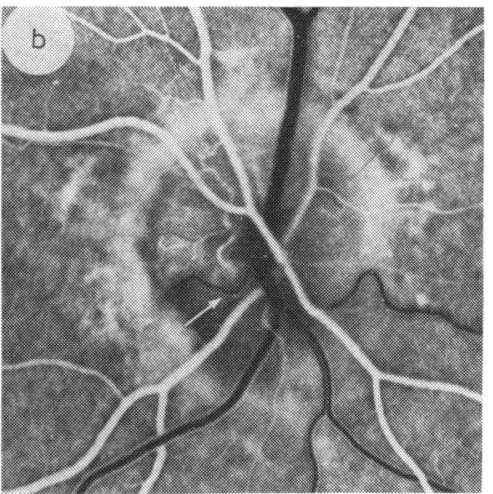

Fig. 1 (Case 1). (a) The right disc has multiple tortuous optociliary veins inferiorly and at 2 and 10 o'clock. Note the loss of nerve fibres and the blurred disc margins. (b and c) Fluorescein angiograms of early and late arteriovenous stages of left eye. The small central shunts fill very early. Note the laminar flow pattern (arrow) proving filling from vein.

examinations were normal. Visual acuity was $6 / 4 \cdot 5$, J1 in each eye. Pupillary light reflexes were normal. The blind spots were enlarged bilaterally, and there was a mild general depression of both visual fields. Bilateral papilloedema was present. Cerebrospinal fluid pressure was measured at 360 $\mathrm{mm}$ water. All laboratory and radiological studies, including computerised tomography, were normal. A diagnosis of benign intracranial hypertension was made. Fundus photography was performed 2 years later, at which time optociliary shunt vessels were noted. At this time the cerebrospinal fluid pressure was measured at $250 \mathrm{~mm}$ water.

CASE 5

A 33-year-old male was evaluated for visual loss in the right eye of 2 years' duration. General physical and neurological examinations were normal. Visual acuity was no light perception in the right eye and $6 / 5$, N5 in the left. The visual field of the left eye showed an inferior defect and constriction of all isoptres. There was bilateral papilloedema. The discs were pale, and optociliary shunt vessels were present on the left. Radiological studies were normal, save for an enlarged sella. Computerised tomography showed the ventricles to be of normal size. The optic nerves were curved in the orbits 

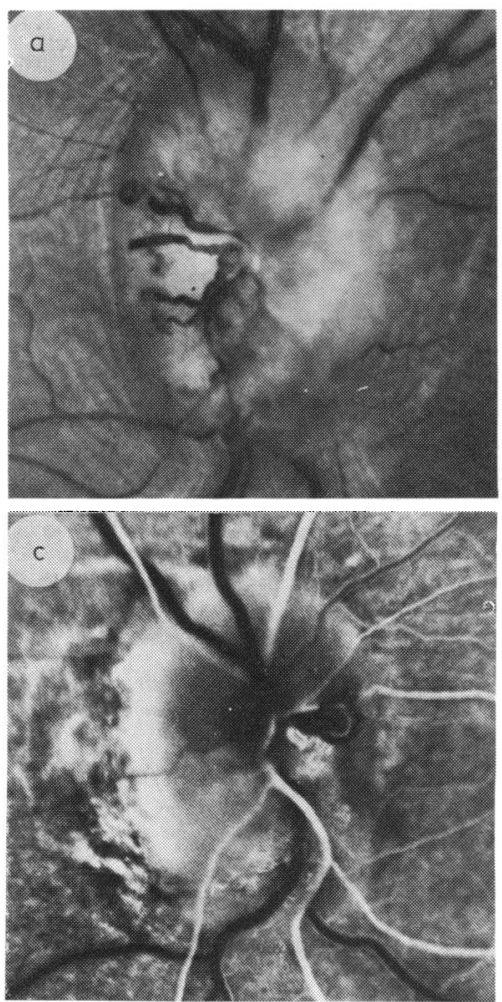

compatible with chronic papilloedema. No abnormality was seen after Conray administration. A diagnosis of benign intracranial hypertension was made.

\section{CASE 6}

A 25-year-old female was evaluated for headaches of 1 year's duration. One year prior to the onset of headaches she began taking the contraceptive pill. General physical and neurological examinations were normal. Vision was 6.5-2 in each eye, and bilateral, severe papilloedema was present. Cerebrospinal fluid pressure was $400 \mathrm{~mm}$ water. Laboratory and radiological studies were all negative. Benign intracranial hypertension was diagnosed and she was treated with steroids. Two and a half years later the left eye had developed a pupillary afferent defect and the vision had declined to 6/12. The left disc showed papilloedema and shunt vessels. The right disc was normal. A lumboperitoneal shunt was inserted.

\section{CASE 7}

A 4-year-old boy was seen with bilateral visual deterioration. The boy had Crouzon's disease, and at the age of 2 years had a repair of his cleft palate. On examination he had the characteristic facial

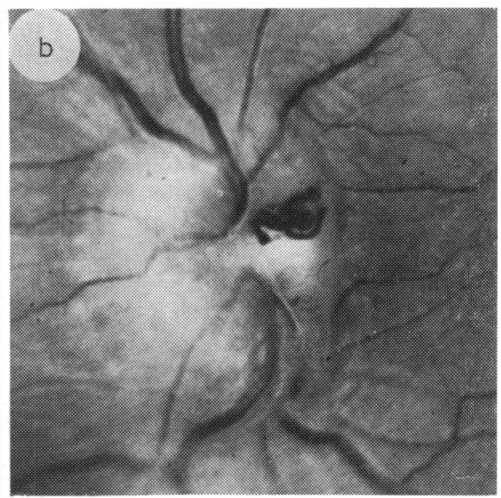

Fig. 2 (Case 2). (a and b) Right and left disc respectively with multiple ectatic and tortuous optociliary veins. Note the retinal and choroidal striae, blurred disc margins, and obscuration of blood vessels on the disc. (c) Fluorescein angiogram of left eye in early arteriovenous stage, showing a laminar flow pattern within the shunt vessel.

appearances of Crouzon's disease, and in addition there were marked epicanthic folds, the bridge of the nose was underdeveloped, and the orbits were shallow. Vision was hard to assess, but he had vague perception of light on the right and he could see objects and fingers with the left. There was a right relative afferent pupillary defect and ocular movements were full. The right optic disc was grossly pale with multiple shunt optociliary vessels (Fig. 4), and the left disc was slightly pale with several shunt vessels. The child was thought to have raised intracranial pressure with Crouzon's disease.

\section{Discussion}

Optociliary shunts may be classified as congenital ${ }^{9} 19$ or acquired. The congenital type shows an anomalous arrangement of the retinal veins, whereas acquired shunts are ectatic and tortuous. Histological studies ${ }^{24}$ have shown that acquired optociliary shunts connect the retinal venous system to that of the choroid. The laminar flow pattern on fluorescein angiograms conclusively shows that these vessels fill from the retinal venous system. The capillary plexus of the prelaminar region of the disc has its arterial and venous connections largely to 


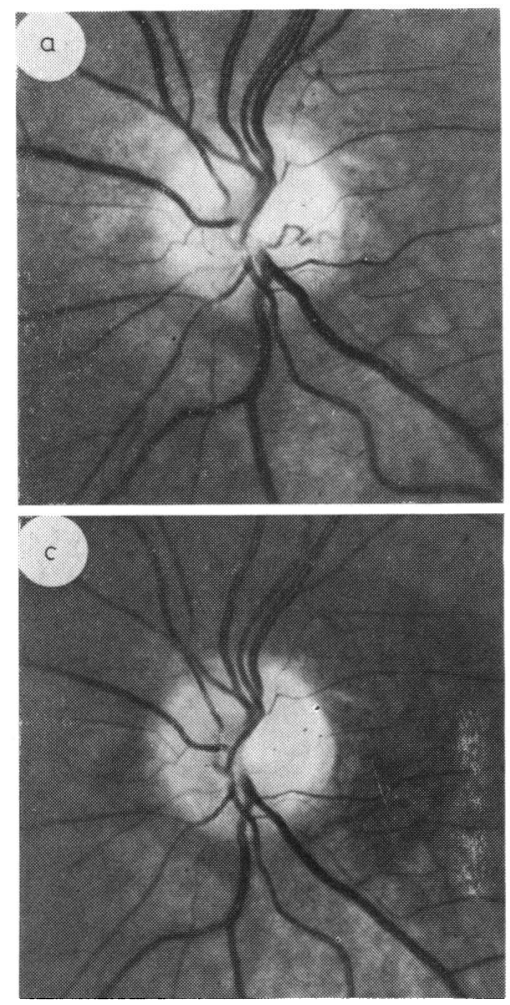

the adjacent choroid. ${ }^{2021}$ However, the capillaries of the disc are all interconnected. The central retinal vein communicates with the choroidal circulation in the prelaminar region. Acquired shunts are con-

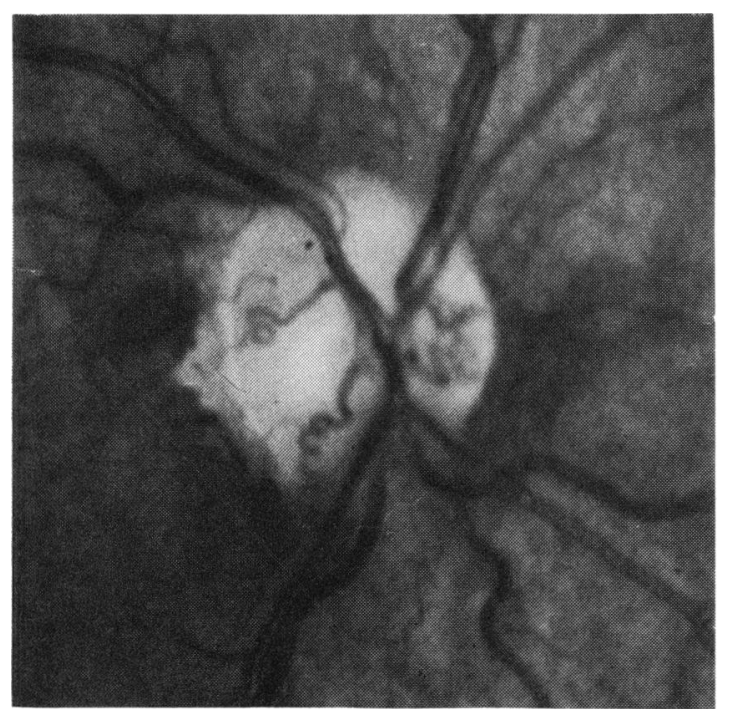

Fig. 4 (Case 7). Right disc with multiple tortuous optociliary shunts and optic atrophy.

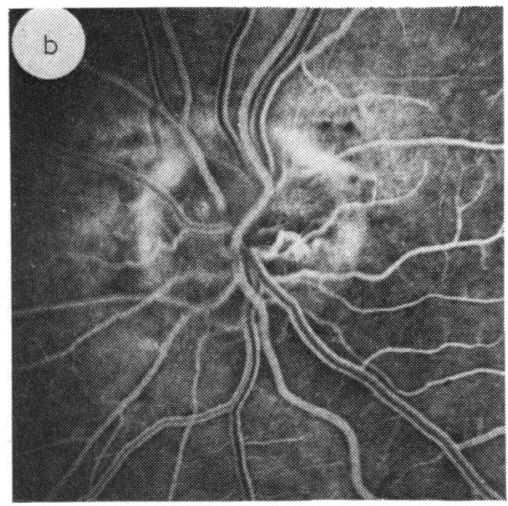

Fig. 3 (Case 3). (a) Left disc with temporal optociliary shunt and optic atrophy. (b) Fluorescein angiogram shows filling of shunt from retinal vein. (c) Left disc 11 days after surgery shows no trace of the shunt vessel.

ceived as forming through the gradual dilatation and enlargement of anastomotic capillary channels with gradual compression or strangulation of the distal optic nerve sheath and central retinal vein by a tumour or the effects of raised cerebrospinal fluid pressure in papilloedema.

Any optic nerve tumour which is situated sufficiently far forward to obstruct retinal venous drainage directly or through chronic disc oedema would be expected to produce optociliary shunts, given a sufficiently long period of time. It has been shown ${ }^{20}$ that obstruction of the central retinal vein by itself is insufficient to produce disc oedema. However, the formation of optociliary shunts in papilloedema provides evidence that there is, at least in some cases, an elevation of central retinal venous pressure. It is of interest that in case 3 the shunt vessel disappeared after surgical relief of intracranial pressure, implying its dependence on elevated central retinal venous pressure to remain open.

Each of the patients described above had papilloedema for a prolonged period of time, 2 with intracranial tumours, 4 with benign intracranial hypertension, and 1 with Crouzon's disease. This prolonged oedema may lead to certain changes in the disc tissue, which are referred to as vintage 
papilloedema, ${ }^{17}$ and eventually the disc may become atrophic. Vintage papilloedema is characterised by shunt vessels and drusen-like bodies at the disc surface or margin which are thought to represent nerve fibre degeneration. Cases 3,5 , and 7 have progressed beyond vintage papilloedema to atrophy with severe visual loss, the shunt vessels remaining as evidence of the preceding prolonged oedema. The triad of tortuous optociliary shunt vessels, prolonged blindness, and a poorly demarcated, atrophic disc may thus arise from papilloedema as well as optic nerve tumour. This triad should not be regarded as specific for optic nerve or sphenoorbital meningioma. ${ }^{1}$ These findings were also present in a case of optic nerve arachnoid cyst, ${ }^{6}$ and we have seen optociliary shunts in longstanding cavernous haemangiomas of the orbit and with optic nerve gliomas.

The authors are grateful to Mr R. Sennhenn for photographic work and Mrs G. Jurewicz for secretarial assistance.

This study was supported in part by a fellowship grant from Columbia University (H. Eggers).

\section{References}

${ }^{1}$ Frisen L, Hoyt WF, Tengroth BM. Optociliary veins, disc pallor and visual loss: a triad of signs indicating sphenoorbital meningioma. Acta Ophthalmol (Kbh) 1973; 51: 241-9.

${ }^{2}$ Rodrigues MM, Savino PJ, Schatz NJ. Spheno-Orbital Meningioma. Am J Ophthalmol 1975; 81: 666.

${ }^{3}$ Ellenberger CC. Perioptic Meningiomas. Arch Neurol 1976; 33: 671-674.

${ }^{4}$ Salzman M. Zur Anatomie der Angeborenen Sichel nach innen-unten. Albrecht von Graefes Arch Klin Ophthalmol 1893; 39: 131-150.

${ }^{5}$ Hoyt WF, Beeston D. The Ocular Fundus in Neurologic Disease. St. Louis: Mosby, 1966.

${ }^{6}$ Miller NR, Green WR. Arachnoid cysts involving a Portion of the intraorbital optic nerve. Arch Ophthalmol 1975; 93: 1117-1121.

${ }^{7}$ Karel J, Otradovek J, Peleska M. Fluorescence angiography in circulatory disturbances in drusen of the optic disk. Ophthalmologica 1972; 164: 449-62.

${ }^{8}$ Stellwag C von C. Lehrbuch der praktischen Augenheilkunde, 2nd ed. Vienna: Braunmüller, 864; 283.

${ }^{9} \mathrm{Kraupa}$ E. Die Anastomosen an Papillen-und Netzhautvenen. Arch Augenheilk 1915; 78: 182-207.

${ }^{10}$ Hitchings RA, Spaeth GL. Chronic Retinal Vein Occlusion in Glaucoma. Br J Ophthalmol 1976; 60: 694-699.

${ }^{11}$ Oosterhuis JA. Fluorescein fundus photography in retinal vein occlusion. In: Henkes HE, ed. Perspectives in Ophthalmology. Amsterdam: Excerpta Medica, 1968; 29-47.

${ }^{12}$ Larsen HW. Manual and Colour Atlas of the Ocular Fundus. Copenhagen: Munksgaard, 1969; 198.

${ }^{13}$ Kohner E, Shilling J. In Rose C, ed. Medical Ophthalmology. Chapman and Hall, London: 1976; chapter 28.

${ }^{14}$ Liebreich R. Atlas der Ophthalmoskopie, 1st ed. Berlin: A. Hirschwald, 1863; PI XI, Fig 8.

${ }^{15}$ Vossius A. Lehrbuch der Augenheilkunde, 2nd ed. Leipzig: Denticke, 1892; 583.

${ }^{16}$ Paton L, Holmes G. The pathology of papilloedema. Brain, 1911 ; 33: 389-432.

${ }^{17}$ Sanders MD. A Classification of papilloedema based on a fluorescein angiographic study of 69 cases. Trans Ophthalmol Soc UK 1969; 89: 177-192.

${ }^{18}$ Sanders MD. Foster Kennedy Sign. Proc R Soc Med 1972; 55: 520-522.

${ }^{19}$ Braune. Ein Beitrag zur Kenntnis optikociliorer Gefasse. Klin Monatsbl Augenheilk 1905; 43: 579-588.

${ }^{20}$ Hayreh SS. Pathogenesis of oedema of the optic disc. Doc Ophthalmol 1968; 24: 289-411.

${ }^{21}$ Hayreh SS. Blood Supply of the optic nerve head and its role in optic atrophy, glaucoma and oedema of the optic disc. $B r J$ Ophthalmol 53: 721-748. 